\title{
Simplified method for the measurement of segmental colonic transit time
}

\author{
A Notghi, R Hutchinson, D Kumar, N B Smith, L K Harding
}

\begin{abstract}
Segmental colonic transit has been measured in 101 patients. Two MBq of ${ }^{111}$ Indium absorbed on resin pellets and encapsulated in an enteric coated capsule was given at 700 am. Hourly images during the first day, and three images during each subsequent day were acquired for up to three days. Using all scan and patient data the scans were categorised in one of the five patterns of colonic transit: normal, rapid, right delay, left delay, or generalised delay. The geometric centres and per cent activity at each time point was compared between the five groups of colonic transit patients to find the best time for imaging and so to distinguish the five groups. During the first day, early images did not help in diagnosis of patterns of transit, however, in the later images (six hours onwards after the ingestion of the activity) the rapid transit groups could be identified. Images at 27 and 51 hours were both required to distinguish all five groups of patients from each other. Only in the 'normal' transit patients was there some excretion of the activity during the course of the second day, otherwise there was no difference in the images taken in the course of a day (second or third day). A simplified protocol requires a minimum of three images to distinguish all five patterns of colonic transit. The activity should be ingested in the morning $(700$ am) and the first image taken at the end of the working day (8-10 hours after ingestion), the second image on the morning of the second day, and the third image during the course of the third day. This simple protocol would provide all the clinically relevant information necessary for correct classification of the colonic transit.
\end{abstract}

(Gut 1994; 35: 976-981) and Nuclear Medicine, Dudley Road Hospital, Birmingh

L K Harding

\section{Department of} Surgery, Queen Elizabeth Hospital, Birmingham $R$ Hutchinson D Kumar

N B Smith

Correspondence to: Dr A Notghi, Department of Physics and Nuclear Physics and Nuclear Medicine, Dudley Road Hospital, Dudley Road, Birmingham B17.

Accepted for publication 10 November 1993 Radiology has established itself as the main method of measurement of colonic transit. Elaborate $x$ ray techniques, however, have been abandoned in favour of simplified tests for routine clinical use. ${ }^{4}$ Scintigraphy is one of the least invasive of these procedures, and yet can supply clinicians with important functional

There has been an increasing interest bowel function using manometric, electrophysiological, volumetric, $x$ ray, and scintigraphic methods, some of which are invasive information. ${ }^{56}$ As with many new investigations the early protocols entail extensive monitoring over prolonged time to obtain the fullest possible information. ${ }^{57}$ Krevsky et al has instilled radioactivity directly into the caecum. ${ }^{7}$ This permits detailed and accurate measurement of the transit through the colon. It may not be entirely physiological, however, because of intubation and it is not practical for routine clinical use. Most other studies 568 have used multiple imaging during the first day followed by several images in the following three to seven days. Some centres have already used a simplified method for clinical practice, ${ }^{9}$ but there is no study that evaluates and rationalises the use of this simplified method for routine colonic transit study. We have analysed our detailed information on colonic transit in different clinical situations with up to 70 images taken over three days. We have then reviewed all the information obtained from such detailed studies to determine the optimal number and timing of images so that the minimum number of images can be acquired with no loss of diagnostic accuracy. This has permitted us to devise a simple procedure that provides the required clinical information. We hope this simple test will permit most nuclear medicine departments to offer colonic transit assessment as a routine service.

\section{Patients and methods}

One hundred and twenty patients received colonic transit studies as part of a detailed investigation for gastrointestinal disorders. These patients included 47 with idiopathic chronic constipation, 27 with faecal incontinence, 5 with diabetic enteroparesis, 25 with irritable bowel syndrome, 8 postabdominal surgery, and 8 normal subjects. Nineteen patients had incomplete clinical or scintigraphic data and were therefore excluded from this analysis leaving 101 patients in the study. Their mean age was 44 years (SD 19) with an age range of 6 to 89 years. Eighty were women $(80 \%)$ and 21 men $(20 \%)$. None of the subjects was taking any drugs likely to affect gastrointestinal transit. All studies were performed on an unprepared bowel. The subjects were asked to continue with their normal diet and activity for the duration of the test. They were in particular asked to avoid laxatives, hot and spicy food, and excessive alcohol. They were also asked to continue with their normal activities avoiding any unusual exercise.

${ }^{111}$ In was absorbed on to resin pellets and encapsulated in a $\mathrm{pH}$ sensitive coated capsule. ${ }^{51011}$ This was designed to release the activity in the ileum. The capsule was given 
between 5 and 7 am and imaging started at 9 am. Anterior and posterior images were acquired at least hourly during the first day until $5 \mathrm{pm}$. A minimum of three sets of images were obtained during the following two days: morning, noon, and afternoon. The investigation was stopped at the end of the third day. On average 14 pairs of anterior/posterior indium images and 14 pairs of marker images were acquired during the three days. Images of cobalt markers on iliac crests were used to align the colonic images. Four regions of interest were drawn over the colon: 1: caecum, ascending colon, and hepatic flexure; 2 : transverse colon; 3: splenic flexure; 4: descending colon to rectum. The activity in each region at each time point was calculated after counts had been corrected for background and decay. The percentage of activity in each region of colon was calculated from the geometric mean of the anterior and posterior images. Geometric mean was used to give a count independent of depth within the body. ${ }^{12} \mathrm{~A}$ fall in the geometric mean of the whole field counts was assumed to result from faecal loss (region 5). The detailed method has been described previously. ${ }^{11}$ The geometric centre ${ }^{367}$ at each time point was calculated using the following formula:

$$
\mathrm{GC}=\Sigma_{\mathrm{n}}^{1} \frac{\% \text { activity in region } \mathrm{n} \times \mathrm{n}}{100}
$$

Parametric images were developed for all patients using the per cent activity in each region. In the parametric images time after ingestion was plotted on the $x$ axis and the percentage of activity in each region ( 1 to 5 ) is shown by a grey scale on the $y$ axis. The full method of parametric image production is described previously. ${ }^{11}$ In addition, the time of arrival at each region and time of peak in the caecum were calculated using the raw images and per cent activity in each region. The time of arrival was assumed to be the first frame when the activity is seen in the region of interest ( $<10 \%$ of administered activity). All time points were calculated from the time of ingestion of the capsule (time 0 ).

Two independent observers reviewed all the results. In particular, parametric images were found to be most helpful in reaching a decision about pattern of transit in these patients. Five distinct types were identified: (1) 'Normal' colonic transit (normal) $(n=36)$ : the most common pattern. These patients had all excreted the activity by the end of the three day study. None had been excreted within the first 10 hours of the study; (2) Generalised delay (G delay) $(n=16)$ : no activity was excreted by the end of the study. The activity was more or less evenly distributed with no specific focal point of accumulation; (3) Right sided delay ( $R$ delay) $(n=32)$ : no activity was excreted by the end of the study. The activity accumulated in regions one and two and stayed there for the length of the study; (4) Left sided delay (L delay) ( $n=4)$ : no activity was excreted by the end of the study. The activity moved along the colon as in the 'normal' group but stayed in region 4 (descending colon/rectum) to the end of the study; (5) Rapid colonic transit (rapid) $(n=13)$ : all the activity was excreted by the morning of the second day. The main distinguishing point from the 'normal' group was that the activity had already moved distally in the colon by the end of the first day. This did not occur with the 'normal' or intermediate group.

The time from ingestion of the capsule to arrival in the caecum (region 1) and each subsequent colonic region was compared among the five groups of patients to find out if these criteria on their own would distinguish the groups. Arrival time was estimated by looking at the analogue images and determining the first frame in which activity could be seen in the region of interest ( $>10 \%$ activity). The time to caecal peak and the time for the activity to appear in faeces was also compared.

A second set of analyses was performed to determine which of the recorded imaging times was the most appropriate to define the five groups of transit patterns clearly so that a simplified method could be based on these images: (1) The percentage of activity in each region of interest was compared between the five groups at $4,6,8,10$ hours after the ingestion on the first day, at $27,31,34$ hours on the second day, and at $51,55,58$ hours on the third day; (2) the geometric centre for each group was also compared at each time point; (3) activity in each region during the course of second and third day (three images each day) was compared to detect any significant difference between images on the same day.

As we rely on the parametric images for routine reporting the adequacy of imaging for the simplified method was assessed by looking at the raw and parametric images at proposed time points: the end of the first day $(10 \cdot 2 \mathrm{SD}$ 1.6 hours after ingestion), on the morning of the second day (27.3 SD $2 \cdot 1$ hours), and the morning of the third day ( $51.8 \mathrm{SD} 1.8$ hours). The ability of these three sets of images in distinguishing the five groups of patients was also tested by comparing the percentage of activity in each region in the five groups and also by calculation of the geometric centres.

The mean and standard deviation (SD) was calculated for each region, for each patient group, and at each time point. Analysis of variance was used to compare several means at each time point. The $F$ test and unpaired Student's $t$ test were used for individual group comparisons.

\section{Results}

\section{PER CENT DISTRIBUTION OF THE ACTIVITY IN}

\section{COLON}

At four hours after ingestion of the capsule less than $50 \%$ of the administered activity was in the caecum in any of the patient groups. The overlapping of the small bowel and terminal ileum activity on the colonic regions 2 and 4 was a potential problem up to six hours after ingestion of the radioactive meal. 


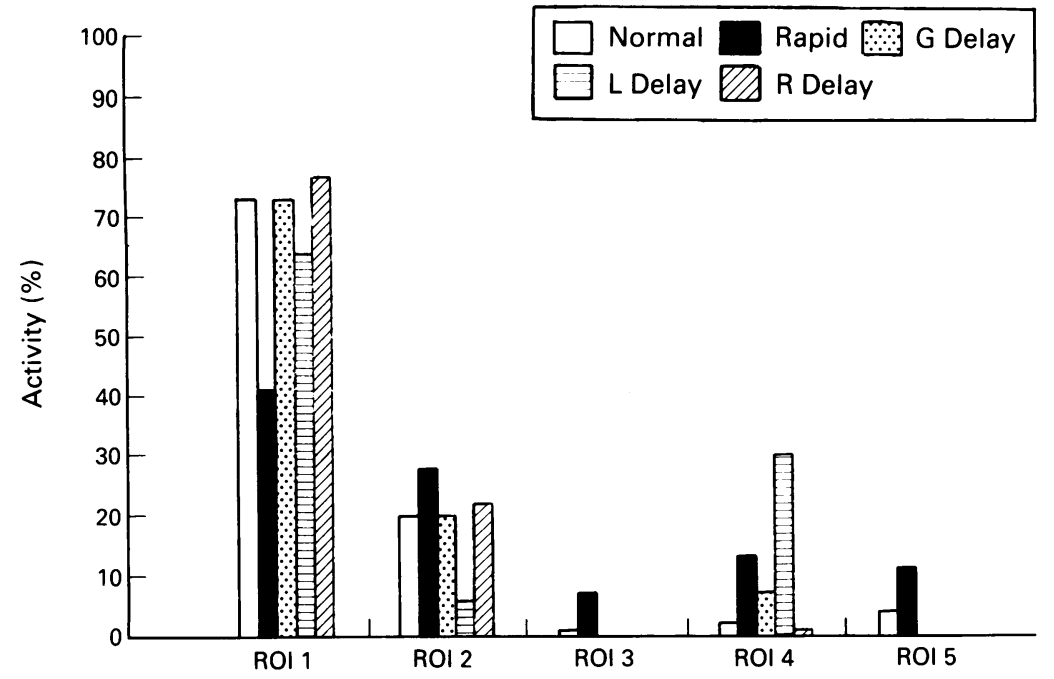

Figure 1: Activity in each colonic region (region 1 ascending colon, 5 faeces) at 10 hours in five groups of patients. Note the wide distribution of activity in patients with rapid transit.

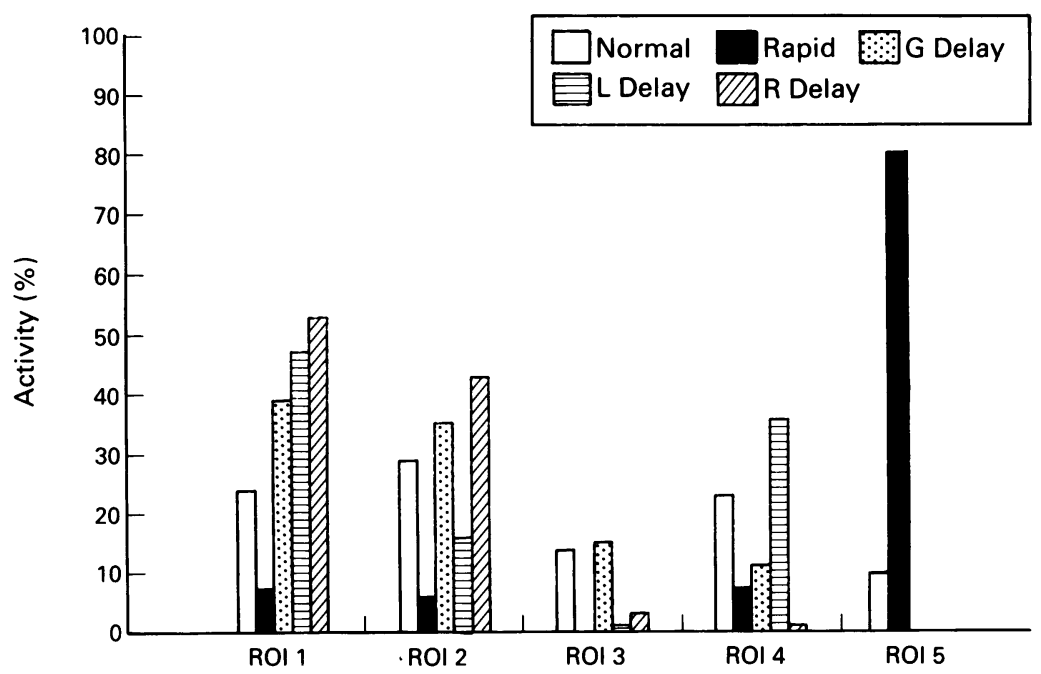

Figure 2: Activity in each colonic region at 27 hours in five groups of patients. Most of the activity is excreted (ROI 5) in rapid transit patients, but none in delayed transit.
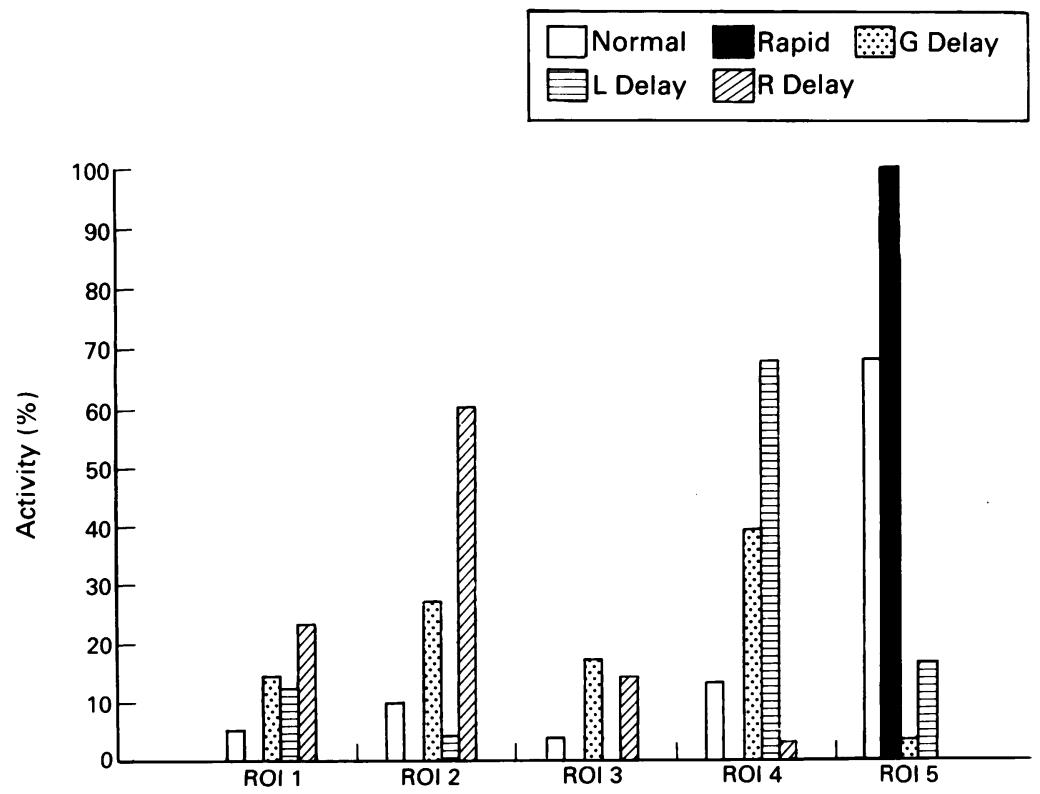

Figure 3: Activity in each colonic region at 51 hours in five groups of patients. There is a different pattern of distribution in each group of patients with delayed transit.
At six hours most of the activity had reached the caecum in all groups, but there was no statistically significant difference between the five groups up to eight hours. From eight hours there were consistent differences between groups, reaching a peak at 10 hours. Ten hour images showed that some of the activity in patients with left sided delay and rapid colonic transit had traversed the colon with significant accumulation in sigmoid colon and rectum (23\% of the activity) in patients with left sided delay, with rapid transit group being the only group with some excretion $(22 \%)$ by this time (Fig 1). There was significant difference between left and right sided delay patients $(p<0.001)$ in region 4 at this stage. Thus the late images in the first day showed increasing difference between $\mathrm{R}$ delay, $\mathrm{L}$ delay, and rapid transit with much overlap between the other groups $(p=N S)$. Figure 1 shows the activity in each region at 10 hours.

During the course of the second day three sets of images were taken $(27,31$, and 34 hour images). At 27 hours (morning image) there was little activity left in the caecum in rapid transit patients (6\% SD 7), while a quarter of the activity was still in the caecum in the normal group (24\% SD 23) (Fig 2). Both these groups were significantly different from any of the delayed transit group $(p<0.001)$. There was no difference, however, among the three groups with delayed transit. Activity in splenic flexure was only seen in the normal and generalised delay groups. The rectosigmoid region contained a third of the activity in the left sided delay, $20 \%$ in the normal, only $10 \%$ in the generalised, and none in the right sided delay (no activity reaches the area), and none in the rapid transit as most of the activity was excreted by 27 hours. Almost all the activity was excreted by 27 hours in rapid transit patients. There was no change in the activity in different regions during the course of the second day $(p=N S)$ for any group of patients except for reduction in the activity in transverse colon and some faecal excretion in patients with normal transit $(\mathrm{p}<0 \cdot 01)$.

At 51 hours (morning of the third day) most of the ingested activity (68\% SD 27) was in the faeces in normal transit group (Fig 3). The remaining activity was mainly in the right colon. The most striking difference was between the normal group and the patients with delayed transit $(p<0.001)$. In $\mathrm{L}$ delay most of the activity concentrated in the sigmoid and rectum (62\% SD 4$)$, in R delay in the right colon $(23 \%$ SD 17 in region 1 and $60 \%$ SD 21 in region 2), and evenly distributed in the $\mathrm{G}$ delay patients. Those with $L$ delay could be distinguished from $G$ delay and $R$ delay by virtual absence of activity in region 1 and 2 . $R$ delay patients had little activity beyond the transverse colon distinguishing this group from those with $G$ delay (Table I). There was no difference in the distribution of activity between the images taken on the third day (images taken at 51,54, and 58 hours after ingestion). Table II lists the significantly different regions at 10,27 , and 51 hour images. 
TABLE I Significance table ( $p$ value) for geometric centres at each time point for each group

\begin{tabular}{|c|c|c|c|c|c|c|c|c|}
\hline & \multicolumn{8}{|c|}{ Imaging hours after ingestion of activity } \\
\hline & \multicolumn{2}{|l|}{ Day 1} & \multicolumn{3}{|l|}{ Day 2} & \multicolumn{3}{|l|}{ Day 3} \\
\hline & 8 & 10 & 27 & 31 & 34 & 51 & 55 & 58 \\
\hline Normal $v$ rapid & NS & NS & 0.001 & 0.05 & 0.005 & NS & NS & - \\
\hline Normal $v \mathrm{G}$ delay & NS & NS & 0.02 & 0.005 & 0.01 & 0.001 & 0.001 & 0.001 \\
\hline Normal $v$ L delay & NS & NS & NS & NS & NS & 005 & 0.02 & NS \\
\hline Normal $v \mathrm{R}$ delay & 0.005 & 0.005 & 0.001 & 0.001 & 0.001 & 0.001 & 0.001 & 0.001 \\
\hline Rapid $v$ G delay & NS & NS & 0.001 & 0.001 & 0.001 & 0.001 & 0.01 & - \\
\hline Rapid $v \mathrm{~L}$ delay & NS & NS & 0.001 & 0.001 & 0.001 & 0.001 & 0.01 & - \\
\hline Rapid $v \overline{\mathbf{R}}$ delay & 0.05 & 0.005 & 0.001 & 0.001 & 0.001 & 0.001 & 0.001 & - \\
\hline $\mathrm{G}$ delay $v \mathrm{~L}$ delay & NS & NS & NS & NS & NS & NS & NS & NS \\
\hline $\mathrm{G}$ delay $v \mathrm{R}$ delay & 0.01 & 0.05 & 0.001 & 0.001 & 0.001 & 0.001 & 0.001 & 0.001 \\
\hline $\mathrm{L}$ delay $v \mathrm{R}$ delay & 0.005 & NS & 0.05 & 0.05 & 0.01 & 0.001 & 0.001 & 0.001 \\
\hline
\end{tabular}

TABLE II The regions of interest at 10,27, and 51 hours that show significant difference in geometric centres $(p<001)$ are listed at the relevant hour

\begin{tabular}{llll}
\hline & 10 Hours & 27 Hours & 51 Hours \\
\hline Normal $v$ Rapid & $\mathrm{R} 1$ & $\mathrm{R} 1-\mathrm{R} 5$ & - \\
Normal $v$ G delay & - & - & $\mathrm{R} 1-\mathrm{R} 5$ \\
Normal $v$ L delay & $\mathrm{R} 4$ & - & $\mathrm{R} 4, \mathrm{R} 5$ \\
Normal $v$ R delay & - & $\mathrm{R} 1, \mathrm{R} 3-\mathrm{R} 5$ & $\mathrm{R} 1-\mathrm{R} 5$ \\
Rapid $v$ G delay & - & $\mathrm{R} 1-\mathrm{R} 3, \mathrm{R} 5$ & $\mathrm{R} 1-\mathrm{R} 5$ \\
Rapid $v$ L delay & - & $\mathrm{R} 1, \mathrm{R} 4, \mathrm{R} 5$ & $\mathrm{R} 1, \mathrm{R} 4, \mathrm{R} 5$ \\
Rapid $v$ R delay & $\mathrm{R} 1, \mathrm{R} 3$ & $\mathrm{R} 1, \mathrm{R} 2, \mathrm{R} 5$ & $\mathrm{R} 1, \mathrm{R} 2, \mathrm{R} 5$ \\
G delay $v$ L delay & - & - & $\mathrm{R} 2, \mathrm{R} 3$ \\
G delay $v$ R delay & $\mathrm{R} 4$ & $\mathrm{R} 3, \mathrm{R} 4$ & $\mathrm{R} 2, \mathrm{R} 4$ \\
L delay $v$ R delay & $\mathrm{R} 4$ & $\mathrm{R} 4$ & $\mathrm{R} 2, \mathrm{R} 4, \mathrm{R} 5$ \\
\hline
\end{tabular}

\section{GEOMETRIC CENTRE}

Geometric centres (GC) were calculated for all the time points for each group of patients. Figure 4 shows the progression of GC through the three days of the study. (The initial apparent high GC in the right sided and generalised transit delay groups results from the overlapping of small bowel activity, which persisted for up to eight hours in some patients.) At 8

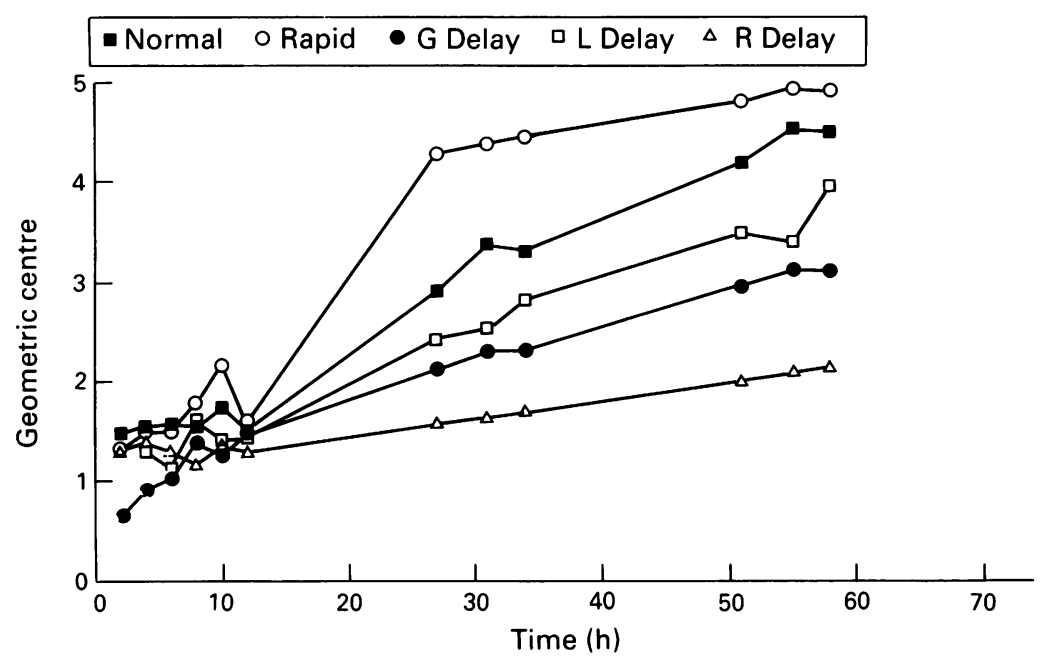

Figure 4: Progression of geometric centres for each group of patients over the course of a three day study. The most clearly distinguishable groups of patients are right sided delay with slow, and rapid transit with fast progression of geometric centres.

TABLE III The mean time (SD) interval (hours) between oral intake and arrival time into each region

\begin{tabular}{|c|c|c|c|c|c|}
\hline & $\begin{array}{l}\text { Region } 1 \\
\text { Caecum }\end{array}$ & $\begin{array}{l}\text { Region } 2 \\
T \text { Colon }\end{array}$ & $\begin{array}{l}\text { Region } 3 \\
\text { S Flexure }\end{array}$ & $\begin{array}{l}\text { Region } 4 \\
\text { Rectum }\end{array}$ & $\begin{array}{l}\text { Region } 5 \\
\text { Faeces }\end{array}$ \\
\hline $\begin{array}{l}\text { Normal } \\
\text { Rapid } \\
\text { G delay } \\
\text { L delay } \\
\mathbf{R} \text { delay }\end{array}$ & $\begin{array}{l}4.7(1.4) \\
4.4(0.9) \\
6.7(5.9) \\
5 \cdot 3(1 \cdot 1) \\
5 \cdot 2(1.4)\end{array}$ & $\begin{array}{l}17 \cdot 4(9 \cdot 7) \\
-\quad 6(8 \cdot 0) \\
\overline{25} \cdot 7(10 \cdot 7)\end{array}$ & $\begin{array}{c}25.9(6 \cdot 2) \\
8 \cdot 7(1.8) \\
32 \cdot 6(8 \cdot 6) \\
- \\
82.5(61.9)\end{array}$ & $\begin{array}{c}35.8(27 \cdot 4) \\
14.1(12.4) \\
39.9(11.9) \\
29.9(3.2) \\
143.8(49.4)\end{array}$ & $\begin{array}{c}44.9(24.4) \\
25.7(5.9) \\
148.3(47.8) \\
141.7(58 \cdot 8) \\
167.2(20.5)\end{array}$ \\
\hline
\end{tabular}

$\mathrm{T}$ colon $=$ transverse colon, $\mathrm{S}$ flexure $=$ splenic flexure . and 10 hours GC was significantly lower in patients with $\mathrm{R}$ delay when compared with the rest of the patients $(p<0.01)$. There was, however, no difference between the other groups of patients. Thus GC did not help to distinguish most of the groups at any of the time points during the first day.

During the second day GC could distinguish all groups from each other except normal $v \mathrm{G}$ delay and $\mathrm{L}$ delay $v \mathrm{G}$ delay or $\mathrm{R}$ delay groups. On the third day it could distinguish between all groups except the normal $v$ rapid transit. There was no difference in the distinguishing power between the images performed during the course of each day. Figure 4 shows the geometric centre progression for each group through the study. Table I summarises the results of the comparisons between the groups.

\section{ARRIVAL TIMES}

Orocaecal transit time ranged from less than two hours to over 10 hours. There was no significant difference in oral to caecal arrival time or to caecal peak between the five diagnostic groups (Table III). The arrival of activity in the transverse colon (region 2) was similar in the normal group and in those with rapid colonic transit, but these were significantly different from right sided $(\mathrm{p}<0.01)$ and generalised delay patients $(p<0 \cdot 002)$. Oral to splenic flexure time distinguished only the patients with rapid transit $(p<0.01)$ from other groups. The transverse colon and splenic flexure were not visualised in patients with $L$ delay. Oral to rectal transit time was the same for generalised delay, left sided delay, and the normal group, but was significantly slow in right sided delay $(p<0.001)$ and fast in rapid transit patients $(p<0.05)$ (Table III). The orofaecal time was significantly different between three groups: rapid transit, normal, or delayed colonic transit $(p<0.001)$, but not between different types of delayed transit. Table III shows the means (SD) for arrival times for all colonic regions.

\section{Discussion}

Colonic disorders are common, and objective assessment of colonic function is made by measurement of colonic transit and contractile activity. The tests used are invasive, expensive, and not practical for routine clinical investigation. ${ }^{2}$ Scintigraphic colonic transit is one of the least invasive procedures and is well tolerated by patients but entails prolonged and frequent imaging. ${ }^{3}$ It uses considerable camera and technician time and therefore is not practical for routine clinical use even in large centres. In all our patients we have used a $\mathrm{pH}$ coated capsule, which releases its contents into the ileum before reaching the caecum. It is fortuitous that activity released in the ileum tends to accumulate in the terminal ileum and then is released into the caecum thus in practice permitting us to perform a test akin to direct bolus delivery into the terminal ileum without the need for intubation. ${ }^{11}$ We have analysed 101 colonic transit studies in a variety 
of colonic disorders and established several patterns of transit. These patterns of colonic transit are useful for classification of colonic transit disorders. Having reviewed all our cases, however, we now propose a simplified method that permits us to reach the same diagnostic accuracy with fewer images obtained over a shorter period of time.

The colon is divided into four regions. The regions were selected so that they represent as large an area as possible and to include fixed anatomical points so that they can be identified confidently in all images. The minimum number of regions that would also be useful clinically was deemed to be the four regions selected. For specific trials, however, dealing with functions of different regions the same principle can be applied. Because of anatomical variation and mobility one of the most difficult regions to identify confidently is the descending colon on its own. Analogue images show that the descending colon is rarely the site of accumulation of any activity (hardly visible except in patients with generalised delay). This is also true of splenic flexure, as suggested by the lack of activity in almost any colonic transit patient (because it is anatomically fixed, however, this region can always be identified). The activity in region 4 thus almost always reflects the activity in the rectum. Thus in left sided delay the problem may lie in the rectum or the pelvic floor physiology. We cannot deal with this problem, however, by solely using our scintigraphic data. Combined manometric, electrophysiological, and proctographic information would be required.

A crude way to look at colonic transit is to measure oral intake to appearance of activity in the faeces, assuming total gastrointestinal transit study reflects colonic transit. Oral to faecal excretion time could distinguish normal, rapid, and delayed transit, but not between different types of delayed transit (Table III). The difficulty with orofaecal or ororectal time is the variation in transit of the activity through the stomach and small bowel. The orocaecal time can vary anything between two and 10 hours with no relation to the colonic transit time, thus reducing the overall sensitivity of this test. The test also does not yield any information about the segmental behaviour of the colon.

In our study there was no consistent or significant difference between the percentage of activity in different patterns of colonic transit in the early frames of the study. The first consistent difference between different groups of patients appeared eight hours after ingestion of the capsule. In the early frames calculation of activity in different regions is difficult for several reasons. Most important is the activity in the terminal ileum, which may overlie colonic regions, in particular region 2 (transverse colon) and region 4 (rectosigmoid region). Early frames reflect the small bowel transit (orocaecal time), which does not necessarily relate to the colonic transit. Some centres use a meal stimulus four to six hours after capsule intake to facilitate ileocaecal delivery of the meal. ${ }^{9}$

Although we did not use such a timed 'push meal' to facilitate ileocaecal transit, we did permit patients a normal lunch at midday, five to eight hours after the ingestion of the capsule. In some patients ileal emptying followed this meal, but in most patients it had already occurred (see Table III for caecal arrival in different groups of patient). At 10 hours, in region 1 (caecum and ascending colon) there was significantly less activity in those with rapid transit compared with normal or delayed transit. In $\mathrm{L}$ delay and rapid transit and some normal patients the activity had already passed through region 1 by this time. This suggests that in the group with left sided delay, passage through small bowel and most of the colon is normal. The percentage of activity in the sigmoid and rectum (region 4) was significantly higher in L delay compared with the other groups. The geometric centre at 10 hours also distinguished $\mathrm{L}$ delay from the other groups. An image at the end of the first day (8-12 hours after ingestion of capsule) distinguished rapid transit and left sided delay from other groups of patients.

Second day imaging started at approximately 27 hours after the ingestion of the activity. The percentage of activity in region 1 (caecum and ascending colon) and region 2 (transverse colon) was significantly less in rapid transit patients compared with any of the other groups of patients. Region 4 (sigmoid and rectum) had less activity in $R$ delay and rapid transit groups when compared with the other groups. Most of the activity was already excreted in patients with rapid transit, the only group that showed significant faecal activity in the morning images of the second day (27 hour). During the course of the day the activity in all groups remained the same except in the normal transit group, where the faecal activity increased. This means an image at any time during the second day of the study would represent the activity for that day except in normal subjects. It is preferable to have an early image on the second day to permit a clearer distinction between the rapid transit and normal groups. Later on most of the activity is excreted in faeces in normal as well as rapid transit patients.

Activity in region 1 on the third day image (51 hours) distinguished delayed transit from normal and rapid patterns. Only $G$ delay and $R$ delay still had significant activity in transverse colon and splenic flexure. There was a significant amount of activity in sigmoid and rectum in L delay (62\% SD 4$)$ and to lesser extent in G delay (39\% SD 17). There was little activity remaining in the colon in the rapid transit and normal groups, while in $\mathbf{R}$ delay hardly any activity had reached the rectum by the third day. Region 5 (faeces) had most of the activity with rapid and normal transit patterns, but very little in any of the groups with delayed colonic transit. During the course of the third day there was no change in the images. Thus the timing of the image in the third day is not important and can be at any time to suit the department's work and the patient's time.

Two images, one on the morning of the second day and one on the third day can distinguish all groups of patients when different 
segments of colonic activity are compared. The rapid and normal transit patients, however, could sometimes be difficult to distinguish from each other using only these two images. A 10 hour image (end of first day) would give additional information, which would help distinguish these two groups.

Using the geometric centre it is not possible to distinguish between $\mathrm{L}$ delay and normal on the first and second day, and also $L$ delay and $G$ delay at any time during the three days of imaging. The images at 27 hours were the most useful to distinguish between the other groups when only geometric centres were used. This implies that the geometric centre is not a sensitive method of identifying different patterns of colonic transit, in particular when an individual patient is assessed, as there is great overlap in between the groups.

In summary, day 3 imaging alone would clearly distinguish between three groups of delayed transit. Imaging on day 2 distinguishes normal and rapid transit groups. At times it is difficult, however, to separate individual patients, particularly normal and rapid transit groups if a 10 hour image does not supplement these data. This is especially true if for any reason the second day image is obtained later in the day rather than early morning, as some normal transit patients excrete activity during the second day imaging.

In conclusion, we propose that after an overnight fast the activity is given early in the morning (between 7 am and 9 am). The patients are then asked to take normal meals. The first image should be obtained at the end of the working day (8-12 hours after the ingestion of the activity). Imaging is repeated the following morning, and if a 10 hour image is not obtained the timing of the second day image will be important (early morning image), but with a 10 hour image it is possible to image the patients at any time during the second day. The third image is obtained during the course of the third day at any convenient time as the pattern of activity does not change during the third day.

An imaging protocol that entails only three images and distinguishes between different patterns of colonic transit is both practical and cost effective. It can be performed in any nuclear medicine department, with little demand on time for the staff or the patient, and with relatively small radiation burden to the patient. We hope the wider availability of a reliable and easy test will help clinicians in the investigation of colonic motility disorders.

Part of the data in this paper has previously been published in Pustract form in Nuclear Medicine Communications 1993; 14: 274 .

1 Chaudhary N, Truelove S. Colonic motility. A critical review of methods and results. Am $\mathcal{F}$ Med 1961; 31 86-106.

2 Pemberton JH, Rath DM, Ilstrup DM. Evaluation and surgical treatment of severe chronic constipation. Ann Surg 1991; 214: 403-13.

$3 \mathrm{Kamm}$ MA. The small intestine and colon: scintigraphic quantitation of motility in health and disease. Eur $\mathcal{F} \mathrm{Nucl}$ Med 1992; 19: 902-12

4 Metcalf AM, Phillips SF, Zinsmeister AR, MaCarty RI Beast RW, Wolff BG. Simplified assessment of segmental Beast RW, Wolff BG. Simplified assessment of
colonic transit. Gastroenterology 1987; 92: 40-7.

5 Proano M Camilleri M, Phillips SF, Brown ML Thomforde GM. Transit of solids through the human colon: regional quantification in the unprepared bowel. colon: regional quantification in the

6 van de Sijp JRM, Kamm MA, Nightingale JMD, Britton KE, Mather SJ, Morris GP, et al. Radioisotope determination of regional colonic transit in severe constipation: comparison with radio-opaque markers. Gut 1993; 34: 402-8.

7 Krevsky B, Malmud LS, D'Ercpe F, Mauer AH, Fisher RS. Colonic transit scintigraphy. A physiologic approach to the quantitative measurement of colonic transit in humans. Gastroenterology 1986; 91: 1102-12.

8 Stivland T, Camilleri M, Vassalo M, Proano M, Rath D, Brown $M$, et al. Scintigraphic measurement of regional Brown M, et al. Scintigraphic measurement of regional gut transit in idiopat

9 Notghi A. Letter from Mayo Clinic. Nucl Med Commun 1993; 14: 618-9.

10 Hesslewood SR, Panagamuwa B, Kumar D, Smith NB, Notghi A, Harding LK. Development of dosage form for measuring colonic transit. I Pharm Pharmacol 1992; 44 (suppl): 1086.

11 Notghi A, Kumar D, Panagamuwa B, Tulley NJ, Hesslewood SR, Harding LK. Measurement of colonic transit time using radionuclide imaging: analysis by condensed iming Nucl Med Commun 1993; 14: 204-11.

12 condensed images. Nucl Med Commun 1993, 14: 204-11. J. Gastric emptying in patients with gastric ulcer. Nucl Med Commun 1982; 3: 192-6. 